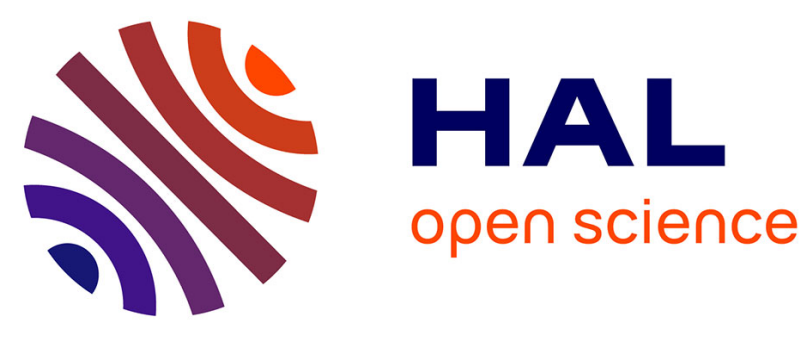

\title{
TRPV4 channels mediate the infrared laser-evoked response in sensory neurons
}

Emmanuel Albert, Jean Michel Bec, Karim Chekroud, Cécile Travo, Sophie

Gaboyard, Gilles Desmadryl, Fabrice Bardin, Isabelle Marc, Michel Dumas, Guy Lenaers, et al.

\section{To cite this version:}

Emmanuel Albert, Jean Michel Bec, Karim Chekroud, Cécile Travo, Sophie Gaboyard, et al.. TRPV4 channels mediate the infrared laser-evoked response in sensory neurons. Journal of Neurophysiology, 2012, 107 (12), pp.3227-3234. 10.1152/jn.00424.2011 . hal-01398845

\section{HAL Id: hal-01398845 \\ https://hal.science/hal-01398845}

Submitted on 13 Mar 2020

HAL is a multi-disciplinary open access archive for the deposit and dissemination of scientific research documents, whether they are published or not. The documents may come from teaching and research institutions in France or abroad, or from public or private research centers.
L'archive ouverte pluridisciplinaire HAL, est destinée au dépôt et à la diffusion de documents scientifiques de niveau recherche, publiés ou non, émanant des établissements d'enseignement et de recherche français ou étrangers, des laboratoires publics ou privés. 


\title{
TRPV4 channels mediate the infrared laser-evoked response in sensory neurons
}

\author{
E. S. Albert, ${ }^{1}$ J. M. Bec, ${ }^{2}$ G. Desmadryl, ${ }^{1}$ K. Chekroud, ${ }^{1}$ C. Travo, ${ }^{1}$ S. Gaboyard, ${ }^{1}$ F. Bardin,,${ }^{2,3}$ \\ I. Marc, ${ }^{2,4}$ M. Dumas, ${ }^{2}$ G. Lenaers, ${ }^{1}$ C. Hamel, ${ }^{1}$ A. Muller, ${ }^{1}$ and C. Chabbert ${ }^{1}$ \\ ${ }^{1}$ U-1051, INSERM Institut des Neurosciences de Montpellier, Montpellier, France; ${ }^{2}$ Université Montpellier 1 et 2 , \\ Montpellier, France; ${ }^{3}$ Université de Nîmes, Nîmes, France; and ${ }^{4}$ Ecole des Mines d'Alès, Alès, France
}

\begin{abstract}
TRPV4 channels mediate the infrared laser-evoked response in sensory neurons. J Neurophysiol 107: 3227-3234, 2012. First published March 21, 2012; doi:10.1152/jn.00424.2011._Infrared laser irradiation has been established as an appropriate stimulus for primary sensory neurons under conditions where sensory receptor cells are impaired or lost. Yet, development of clinical applications has been impeded by lack of information about the molecular mechanisms underlying the laser-induced neural response. Here, we directly address this question through pharmacological characterization of the biological response evoked by midinfrared irradiation of isolated retinal and vestibular ganglion cells from rodents. Whole cell patch-clamp recordings reveal that both voltagegated calcium and sodium channels contribute to the laser-evoked neuronal voltage variations (LEVV). In addition, selective blockade of the LEVV by micromolar concentrations of ruthenium red and RN 1734 identifies thermosensitive transient receptor potential vanilloid channels as the primary effectors of the chain reaction triggered by midinfrared laser irradiation. These results have the potential to facilitate greatly the design of future prosthetic devices aimed at restoring neurosensory capacities in disabled patients.
\end{abstract}

transient receptor potential vanilloid 4; prosthesis

SENSORY PERCEPTION RELIES on the function of highly specialized receptors, which detect a wide range of stimuli arising from our environment and convert them into bioelectrical signals eventually conveyed to the brain. Although highly specialized, the sensory receptors remain fragile, and the loss of these cells through progressive degeneration (Saihan et al. 2009), sudden cell damage, or age-related processes (Chen et al. 2010) cannot be compensated. This has been overcome by the development of sensory prostheses aiming to substitute lacking sensory receptors. Such an approach is only possible if the central sensory neuronal pathway is preserved. In past decades, direct electrical stimulation of the sensory neuronal pathways proved that central sensory input could be generated without stimulation of the sensory receptors. Cochlear implants, for example, were developed as treatment of sensorineural hearing loss, using electrical stimulation of the auditory nerve (Haynes et al. 2009). Through the years, limitations and constraints such as electrode deterioration and poor resolution of environmental sound or visual perception of the existing prostheses have been revealed. Prolonged use of electrodes turned out to be noxious, leading to neural tissue damage and possible toxicity of electrode materials (Wells et al. 2007a). Graded electrical current spread is also observed well beyond the electrode location

Address for reprint requests and other correspondence: C. Chabbert, INSERM U-1051, Hopital St. Eloi, 80 rue A. Fliche, Montpellier 34090, France (e-mail: christian.chabbert@inserm.fr).
(Popovic et al. 1991). In addition, investigations into the safety of retinal electrical stimulation showed that direct tissue contact led to important retinal damage (Colodetti et al. 2007).

Over the years, infrared (IR) laser focal irradiation, which does not require direct contact between the stimulus source and the tissue, has been considered as an alternative approach to stimulate primary sensory neurons under conditions with sensory receptor cell deficits. In the 1970s, Fork (1971) first demonstrated that laser emissions in the blue and green spectrum as well as near-IR wavelengths could be used selectively to stimulate photosensitive neurons from the abdominal ganglion of marine molluscs. Later, it was demonstrated that transient variations of the membrane potential could be evoked through IR laser stimulations on nonphotosensitive neurons such as those of the auditory nerves (Izzo et al. 2006, 2008), cavernous nerve of the prostate (Fried et al. 2008), and facial nerve (Teudt et al. 2007) without tissue damage. High spatial resolution of the stimulus is a further advantage of optical radiation over electrical stimulation.

Although promising, clinical developments of such approaches have been impeded by the lack of information on the molecular mechanisms supporting the laser-evoked neural response. According to Wells et al. (2005), photochemical effects are not involved in mid-IR optical stimulation, whereas photomechanical effects like pressure and stretching cannot occur when operating with short laser pulses at high energies. More likely, transient thermal variations at the cell membrane would be sufficient to trigger laser-induced biological responses. To address this question directly, we undertook a pharmacological characterization of the neural response evoked by mid-IR $(1,875 \mathrm{~mm})$ irradiation in retinal and vestibular ganglion cells (VGCs) isolated from rodents using whole cell patch-clamp recording. Here, we show that both types of neurons can be stimulated reproducibly and that neuronal responses are driven through transient receptor potential vanilloid 4 (TRPV4) channels.

\section{MATERIALS AND METHODS}

Animals. Mice of the C57BL/6 strain and Wistar rats (Centre d'Elevage Janvier, Le Genest-Saint-Isle, France) were used in the study. All procedures were carried out in accordance with the French/ European Communities Council Directive 86/609/EEC.

Cell preparations. Retinal ganglion cells (RGCs) were isolated from postnatal days 2-4 (P2-P4) mice using the Thy-1 antibodymediated plate adhesion immunopanning technique, and their specificity was confirmed using RGC Brn3a immunoassay according to previously described procedures (Kamei et al. 2005). VGCs were explanted from P5-P9 rats and dissociated according to previously described procedures (Dayanithi et al. 2007). 
Electrophysiological recordings. Whole cell patch-clamp recordings (current-clamp configuration) were performed at room temperature $\left(22-24^{\circ} \mathrm{C}\right)$ using a computer-controlled Axopatch 200B amplifier (Axon Instruments, Molecular Devices, Sunnyvale, CA) and digitized using a Digidata 1322A as previously described (Chabbert et al. 2001). Patch electrodes (5-6 M $\Omega$; microhematocrit tubes; Bris Vitrex Medical) were filled with an intracellular solution $(140 \mathrm{mM} \mathrm{KCl}, 15$ $\mathrm{mM} \mathrm{NaC}, 10 \mathrm{mM}$ glucose, $10 \mathrm{mM}$ HEPES, $5 \mathrm{mM}$ EGTA, $3 \mathrm{mM}$ ATP, 1 mM GTP; pH 7.4, 300 mosM). For whole cell recordings, neurons were bathed in extracellular solution: $135 \mathrm{mM} \mathrm{NaCl}, 5 \mathrm{mM} \mathrm{KCl}, 1$

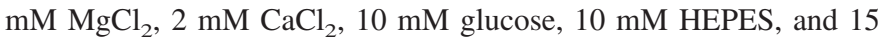
$\mathrm{mM} \mathrm{Na}_{2} \mathrm{HCO}_{3}, \mathrm{pH} 7.4,300$ mosM. Removal of calcium from the bath was performed through local and transient exchange of the bathing solution by calcium-free external solution $\left(0 \mathrm{Ca}^{2+}\right.$ and $2 \mathrm{mM}$ EGTA) through a homemade gravity perfusion system. Studied neurons were first selected on morphological criteria (refringence), resting membrane potential $(-60.0 \pm 5 \mathrm{mV}, n=30 \mathrm{VGCs} ;-35.9 \pm 5 \mathrm{mV}, n=$ 18 RGCs), and excitability [ability to fire action potentials (APs) in response to depolarizing current injections of $200 \mathrm{pA}$ for $1 \mathrm{~s}]$. The lack of cell damage following the IR laser irradiation was determined by the lack of sustained variations of the resting membrane potential, the ability of the cell to exhibit repetitive laser-evoked neuronal voltage variations (LEVV) over minutes, and the ability of the recorded cells to fire APs on injection of depolarizing currents following the pharmacological assessments. For evaluation of each LEVV component, amplitude of the humplike component was determined as the difference between resting membrane potential (mean of $5 \mathrm{~ms}$ before irradiation) and amplitude of the voltage $5 \mathrm{~ms}$ after the onset of the response. Mean peak amplitude of the spikelike component was determined relative to resting membrane potential. In the figures, each trace represents individual record rather than averaged traces.

IR laser stimulation. Sensory neurons were stimulated with a diode laser (Sheaumann Laser, Marlborough, MA) emitting at a wavelength of $1,875 \mathrm{~nm}$. The stimulation system was mounted on an air-cooling block and operated at room temperature. The laser beam was guided through a multimode fiber of $105-\mu \mathrm{m}$ diameter and mounted on a micromanipulator to control fine positioning during the experiments. For better precision and alignment $(165 \pm 10 \mu \mathrm{m})$ to the target cells, visible light was coupled before laser stimulation. A control board (DAQ; PCI-6221; National Instruments) was used to tune all laser-stimulation parameters (radiant exposure, pulse duration, and repetition rate) and to record simultaneously the electrophysiological signals with a LabVIEW program. During all experiments, the optical power at the fiber output was maintained at $230 \mathrm{~mW}$, and the duration of single pulses was increased from 1 to $15 \mathrm{~ms}$ until obtaining of the LEVV. In the two types of sensory neurons, complete LEVV response (including the humplike and the spikelike component; see RESULTS) was always obtained for pulse durations from 7 to $10 \mathrm{~ms}$. This corresponds to a radiant exposure at the output optical fiber ranging from 20 to $60 \mathrm{~J} / \mathrm{cm}^{2}$ (on a circular surface obtained from a radius $\sim 50 \mu \mathrm{m})$.

Estimation of temperature changes yield by the IR laser irradiation. To estimate the temperature changes of the bath, a fluorescent temperature-sensitive dye (LDS 698; Exciton, Dayton, OH) was used as previously described (Coppeta and Rogers 1998; Kim et al. 2009). LDS 698 is active in the range $20-100^{\circ} \mathrm{C}$ and was used at a concentration of $10 \mu \mathrm{M}$. To calibrate the fluorescence intensity changes of the LDS 698 dye on temperature fluxes, the bath without any cells was maintained within a temperature range of $20-50^{\circ} \mathrm{C}$ by a heater controller (Harvard Apparatus, Les Ulis, France). Temperature measurements were carried out in similar conditions as occur during experiments (same fiber position, initial bath temperature, saline bath, and radiant exposure range).

Drugs. TTX, Ni/Cd, $\mathrm{Gd}^{3+}$, and 2-aminoethoxydiphenyl borate (2-ABP) were obtained from Sigma-Aldrich (Lyon, France). Ammoniated ruthenium oxychloride 1430 (ruthenium red), 2,4-dichloro- $N$ isopropyl- $N$-(2-isoopylaminoethyl)benzenesulfonamide (RN 1734), and capsazepine were obtained from Tocris Bioscience (Bristol, United Kingdom). Drugs were applied to the bathing medium in the vicinity of the cell by puff or by using a fast gravity perfusion system.

Real-time PCR assay. Total RNA from isolated cell cultures (RGC and VPN) was extracted by RNeasy Mini Kit (Qiagen, Courtaboeuf, France) according to the manufacturer's protocol. The same amounts of total RNA were employed for first-strand cDNA synthesis by using the Verso cDNA Kit (Thermo Fisher Scientific, Illkirch-Graffenstaden, France). Real-time PCR was performed using LightCycler FastStart DNA MasterPLUS SYBR Green I Kit (Roche Diagnostics, Meylan, France). Amplifications from RGCs (mouse Trpv1, 5'-CATGTCTGGAGCTGTTCAAGTTC-3' and 5'-CGTTGGTGTTCCAGGTAGTCCA-3'; Trpv2, 5'-AGCTGACTGGACTGCTAGAGTAC-3' and 5'TGGATGTGAACATTCGCTCCAT-3'; Trpv3, 5'-TAGGCTCCAGCAATGAATGCCC-3' and 5'-ACACAGCCGCGAAGATGCGCT-3'; TRPV4, 5'-CTATCTGTGTGCCATGGTCATC-3' and 5'-AGAGACAACCACCAGCACAGAG-3'; Actin, 5'-CTGGGCCTCGTCACCCACATA-3' and 5'-GACCCAGATCATGTTTGAGACCTT-3') and VGCs (rat Trpv1, 5'-CATGTCTGGAGCTGTTCAAGTTC-3' and 5'CATTGGTGTTCCAGGTAGTCCA-3'; Trpv2, 5'-AACTGACTGGACTGCTAGAATAC-3' and $5^{\prime}$-GCTCCTCTTCTCTATGGCGATGT-3'; Trpv3, 5'-GCTCCAGCAATGAAAGCCCAC-3' and 5'ATACAGCCGCGAAGATGCACT-3'; Trpv4, 5'-CTATCTGTGTGCCATGGTCATC-3' and 5'-AGACACAACCACCAGCACTGA G-3'; Actin, 5'-CTCTGAACCCTAAGGCCAACC-3' and 5'-GAGTCCATCACAATGCCAGTG-3') were normalized to 18 s ribosomal RNA levels. During the experiments, each sample was investigated in triplicate. The primers have been tested on control tissues (Kunert-Keil et al. 2006) before experiments.

Immunohistochemistry. For in situ experiments, eyes and vestibular ganglions were explanted from adult mice and rats, respectively, and then fixed. For both in situ and in vitro immunostaining, tissues were fixed by immersion for $1 \mathrm{~h}$ in $4 \%$ paraformaldehyde in PBS $(0.1 \mathrm{mM}$, $\mathrm{pH}$ 7.4). Then, in situ samples were embedded in $4 \%$ agarose, and $40-\mu \mathrm{m}$ thick sections were cut using a vibrating blade microtome (Vibratome Series 1000; Technical Products International, St. Louis, MO). Free-floating sections and cultures were then permeabilized in Triton X-100 for $1 \mathrm{~h}, 4 \%$ in PBS for adult tissue, and 1\% in PBS for in vitro samples. A following preincubation in blocking solution ( $0.5 \%$ fish gelatin, 0.5 and $0.1 \%$ Triton $\mathrm{X}-100$ for adult and culture, respectively, in PBS) prevented nonspecific binding. Samples were then incubated with the following primary antibodies: anti-Brn3a (goat polyclonal; dilution 1:200; Sigma-Aldrich), anti-TRPV1 (rabbit polyclonal IgG, 2233; dilution 1:100; Tocris Bioscience), antiTRPV2 (rabbit anti-vanilloid receptor-like protein 1; dilution 1:200; AB5398P; Chemicon International, Millipore, Molsheim, France), anti-TRPV3 (mouse monoclonal; ab85022; dilution 1:100; Abcam), and anti-TRPV4 (rabbit polyclonal; ab39260; dilution 1:200; Abcam). Mouse monoclonal anti-neurofilament $(200 \mathrm{kDa}$, dilution 1:350; clone N52; Sigma-Aldrich, St. Louis, MO), rabbit polyclonal serum anti-TRPV4, and goat polyclonal serum anti-Brn3a were applied for $48 \mathrm{~h}$ rotating at $4^{\circ} \mathrm{C}$ in the corresponding blocking solution. For control, investigated primary antibody was omitted. Secondary antibodies revealed specific labeling with Alexa-conjugated donkey anti-mouse, -rabbit, or -goat sera (1:200; Molecular Probes, Eugene, OR). Samples mounted on slides were observed with a Zeiss 5 LIVE DUO laser-scanning confocal microscope (Zeiss, Le Pecq, France). Human eye-cups obtained from Laboratoire Biologie Ingénierie et Imagerie de la Greffe de Cornée (Saint Etienne, France) were embedded in paraffin and cut into 5 - $\mu \mathrm{m}$ sagittal sections. Sections were deparaffinized, hydrated, and incubated for $20 \mathrm{~min}$ in a saturation buffer (PBS, $0.3 \%$ Triton X-100, 30\% Semliki Forest virus) and overnight with the primary antibody, anti-TRPV4 (rabbit polyclonal, ab39260, 1:200; Abcam). The secondary antibody, Alexa-conjugated donkey anti-rabbit (1:1,000; Molecular Probes), was diluted in PBS and Hoechst and incubated $1 \mathrm{~h}$ at room temperature. Sections were then mounted on slides and observed with a Zeiss ApoTome. 
A

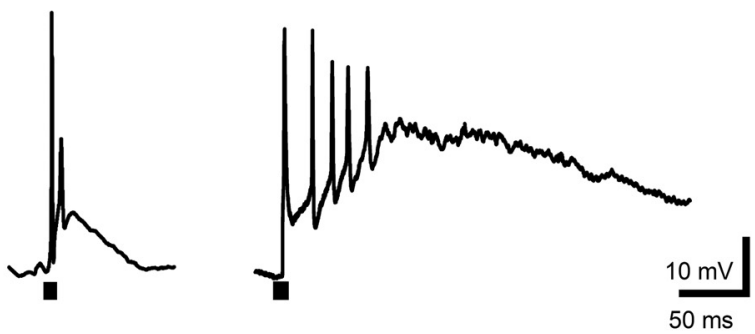

B

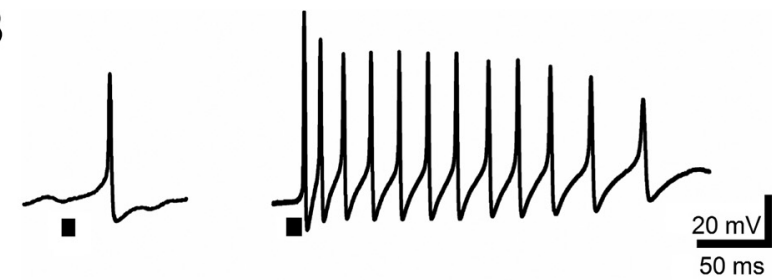

C

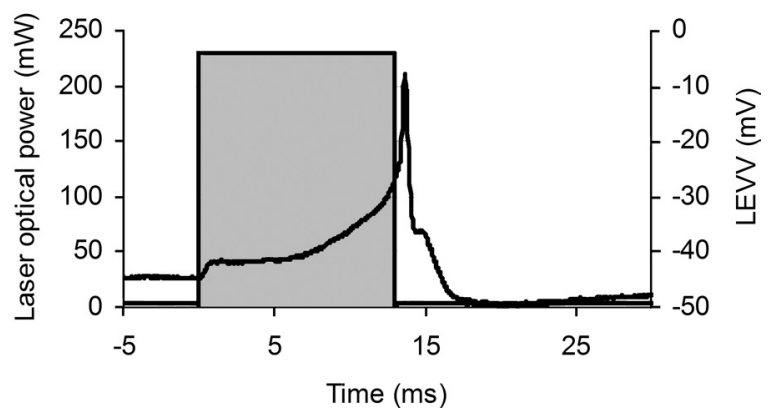

Fig. 1. Midinfrared (mid-IR) laser stimulation induces transient membrane potential variations in sensory neurons. Illustration of phasic (left traces) and tonic (right traces) laser-evoked neuronal voltage variations (LEVV) obtained in 12 retinal ganglion cells (RGCs; $A$ ) and 25 vestibular ganglion cells (VGCs; $B$ ) evoked by 7- and 10-ms laser stimulations (black boxes) at $1,875 \mathrm{~nm}$, respectively. $C$ : illustration of the synchronized acquisition of the laser stimulus (gray area) and the laser-evoked neuronal response that reveals the humplike and spikelike components of the LEVV. Each trace represents individual record.

\section{RESULTS}

Mid-IR laser stimulations trigger transient membrane potential variations in sensory neurons. Isolated RGCs and vestibular primary neurons (VGCs) were irradiated using an 1,875-nm IR laser. LEVVs were obtained in RGCs and VGCs for pulse durations from 7 to $10 \mathrm{~ms}$ at laser power of $230 \mathrm{~mW}$. As illustrated in Fig. 1, either single (phasic) or repetitive (tonic) spike responses could be evoked in RGCs (Fig. 1A) and VGCs (Fig. $1 B$ ) depending on the duration of the irradiation. LEVVs usually displayed an initial humplike depolarization (mean maximum amplitude: $10 \pm 4 \mathrm{mV}, n=12 \mathrm{RGCs}$ and $10 \pm 5 \mathrm{mV}, n=25 \mathrm{VGCs}$ ), on top of which a spikelike component (mean peak amplitude: $30 \pm 5 \mathrm{mV}, n=12 \mathrm{RGCs}$ and $35 \mathrm{mV} \pm 5 \mathrm{mV}, n=25 \mathrm{VGCs}$ ) occurred (Fig. $1 C$ ). The humplike depolarization phase always started within the $1 \mathrm{st} \mathrm{ms}$ after the onset of the irradiation. Conversely, occurrence of the spike component could be delayed up to $10 \mathrm{~ms}$ under our irradiation conditions. LEVVs could be evoked repetitively for several minutes in the two cell types without cell damage. Visual control of the preparation did not reveal any movement of recorded cells nor of the external solution during the irradiation pulses. In a fraction of recorded VGCs $(16 \%, n=$ 4) a small hyperpolarization of $3 \mathrm{~ms}$ preceded the spike component of the LEVV (Fig. 5A). Such hyperpolarization response was never observed in the RGCs.

Transient membrane potential variations rely on voltagegated sodium and calcium channels. To determine the type of ionic channels involved in the generation of RGC and VGC responses, we used several pharmacological reagents. Bath application of TTX $(10 \mu \mathrm{M})$, a specific blocker of the voltagegated sodium channels (Brugeaud et al. 2007), reversibly abolished the spikelike component of the LEVV in the two cell types ( $n=4$ RGCs and $n=7$ VGCs; Fig. $2 A$ ). This effect identified the spikelike component of the LEVV as a sodiumbased AP. Subsequent application of Ni/Cd solution $(500 \mu \mathrm{M})$, a widely used blocker of the low-threshold voltage-gated calcium channels (Chambard et al. 1999), slightly affected (14 $\pm 6 \% ; n=3 \mathrm{RGCs}$ and $n=7 \mathrm{VGCs}$ ) the remaining humplike component of the LEVV (Fig. 2B). Direct application of $\mathrm{Ni} / \mathrm{Cd}$ on the LEVV prevented the generation of the sodium-based AP (Fig. 2C), suggesting that it mostly relied on a calcium influx in the cells through voltage-gated calcium channels. This was confirmed by complete block of the LEVV when calcium was removed from the bathing solution $(n=6$ VGCs; Fig. 2D). Note that residual depolarizing component remained despite removal of external calcium.

A

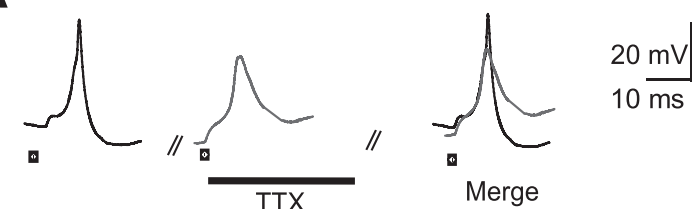

B
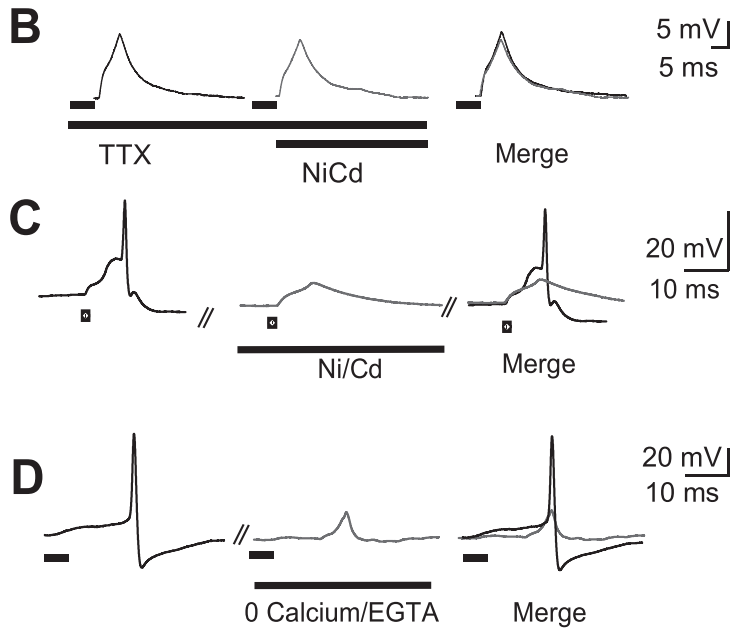

Fig. 2. Ionic components of LEVV evoked in the sensory neurons. Each panel is composed of individual LEVV traces in control saline solution (expected for $B$, left traces) following application of the tested drugs (middle traces) and the merge of control vs. drug tests (right traces). A: effect of bath applications of TTX $(10 \mu \mathrm{M})$ unmasks the TTX-sensitive $\mathrm{Na}^{+}$-mediated component of the LEVV in a RGC ( $n=4$ RGCs and 7 VGCs). $B$ : subsequent bath application of $\mathrm{Ni} / \mathrm{Cd}$ solution $(500 \mu \mathrm{M})$ on a VGC unmasks the small $\mathrm{Ni} / \mathrm{Cd}$-sensitive $\mathrm{Ca}^{2+}$-mediated component of the LEVV and reveal a large TTX- and Ni/Cdresistant component ( $n=3 \mathrm{RGCs}$ and $7 \mathrm{VGCs})$. $C$ : direct application of $\mathrm{Ni} / \mathrm{Cd}$ solution on a VGC suppresses both the $\mathrm{Na}^{+}-$and the $\mathrm{Ca}^{2+}$-mediated components of the LEVV unmasking the residual component ( $n=3 \mathrm{RGCs}$ and 2 VGCs). $D$ : removal of external calcium $\left(0 \mathrm{Ca}^{2+} / 2 \mathrm{mM}\right.$ EGTA) from the bathing solution abolishes the LEVV in a VGC ( $n=6 \mathrm{VGCs})$. Note that a residual depolarizing component remained despite removal of external calcium. Scale in $D$ applies to all panels. 
TRPC channels are not involved in the IR laser-evoked response in sensory neurons. Transient receptor potential canonical (TRPC) channels were previously reported to mediate lightevoked calcium responses in intrinsically photosensitive RGCs (ipRGCs; Hartwick et al. 2007). Bath applications of either 2-APB (100 $\mu \mathrm{M} ; n=2$ RGCs and $n=2$ VGCs) or $\mathrm{Gd}^{3+}$ (10-100 $\mu \mathrm{M} ; n=3$ RGCs and $n=2$ VGCs), two widely used blockers of the TRPC channels, did not affected the LEVV evoked in both cell types (Fig. 3, $A$ and $B$ ). This observation indicated that the TRPC channels were not required for the generation of the LEVV in these sensory neurons.

TRPV4 channels mediate the IR laser-evoked response in sensory neurons. It has been recently proposed that AP generation by pulsed laser stimulation of nerve tissue or neurons is thermally mediated either through thermoradiating or thermomechanical processes (Wells et al. 2007a,b). To test this hypothesis, we investigated whether thermosensitive TRPV channels were involved in the LEVVs evoked in sensory neurons. We first searched for the presence of TRPV1-4 channels mRNA in our preparations of RGC and VGC neurons (Fig. 4). RT-PCR investigations using specific primers (see MATERIALS AND METHODS) revealed that TRPV channel mRNAs were differentially expressed with similar patterns in the two types of sensory neurons. In the RGCs, TRPV2 and TRPV4 mRNAs displayed the major expression, whereas neither the expression of TRPV1 nor TRPV3 was significant (Fig. $4 A$ and $B$ ). Similarly, in VGCs, both TRPV4 and TRPV2 mRNAs displayed high levels of expression, whereas the expression of neither TRPV1 nor TRPV3 mRNAs was significant (Fig. 4, $C$ and $D$ ).

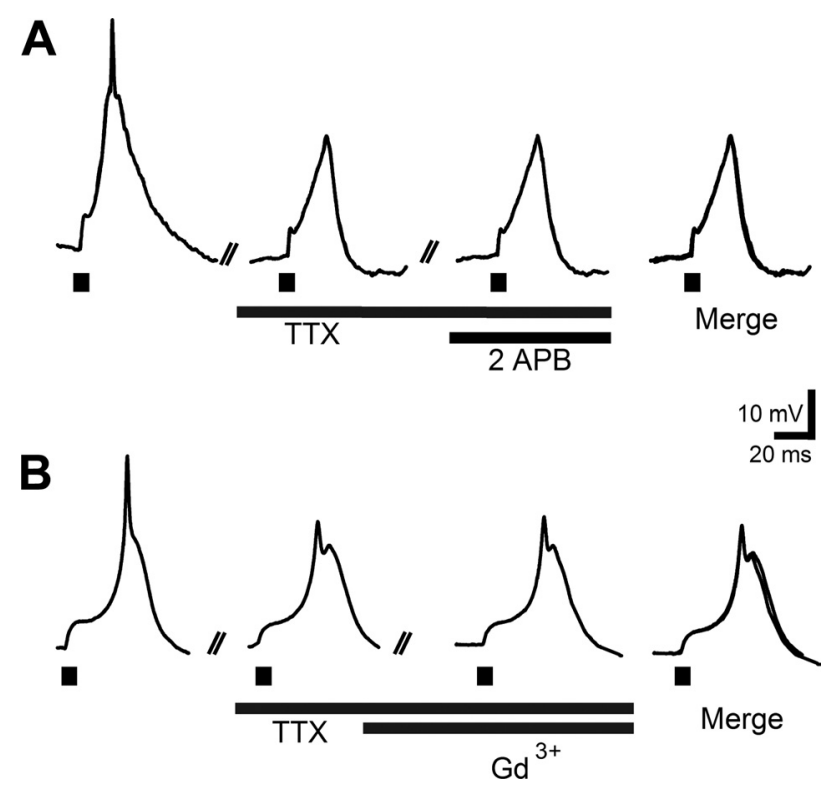

Fig. 3. The transient receptor potential canonical (TRPC) channels are not involved in the generation of the LEVV in the RGCs and the VGCs. Each panel is composed of LEVV traces in control saline solution (left traces) following application of the tested drugs (middle traces) and the merge of TTX-resistant vs. 2-aminoethoxydiphenyl borate (2-ABP) or $\mathrm{Gd}^{3+}$ tests, respectively (right traces). A: application of 2 -ABP $(100 \mu \mathrm{M})$, a selective TRPC channel antagonist, subsequently to TTX $(10 \mu \mathrm{M})$ does not affect the residual, RGC component of the LEVV evoked in a RGC $(n=2$ RGCs and 2 VGCs). $B$ : gadolinium $(10$ and $100 \mu \mathrm{M})$ does not affect the residual component of the LEVV in a VGC ( $n=3$ RGCs and 2 VGCs). Note that in that specific recording, the remaining spikelike deflection despite the presence of TTX may result from a noncomplete block of the voltage-gated sodium channel population.
Pharmacological investigations were subsequently conducted to test for the direct involvement in the LEVV of the TRPV channel subtypes expressed in the RGCs and VGCs. Bath applications of ruthenium red $(10 \mu \mathrm{M})$, a general TRPV channel blocker, completely abolished the LEVV evoked in both the RGCs $(n=3)$ and VGCs $(n=5$; Fig. $5 A)$. This indicated that TRPV channels mediated the LEVV in sensory neurons. Capsazepine $(10 \mu \mathrm{M})$, a specific TRPV1 channel blocker, did not affect the LEVV evoked in the RGCs $(n=2)$ and in the VGCs $(n=4$; Fig. $5 B)$, consistent with the nonsignificant expression levels of TRPV1 in both neuron types (Fig. 4). Conversely, RN 1734 (3.2 $\mu \mathrm{M}$ in VGC and 5.2 $\mu \mathrm{M}$ in RGCs), a specific TRPV4 channel blocker (Angelico and Testa 2010; Vincent et al. 2009), prevented the induction of LEVV in both RGCs $(n=4$; Fig. $5 C)$ and VGCs $(n=7$; Fig. 5D). Altogether, these results indicated that the TRPV4 channels mediate the LEVV in the two types of sensory neurons. The electrically evoked neural responses were not affected on application of ruthenium red or RN 1734 (data not shown). In our hands, SKF-96365 given as a potent TRPV2 blocker (Vriens et al. 2009) significantly altered the voltage-induced excitability of the VGCs, preventing any investigation of the involvement of TRPV2 into the LEVV in this neuronal type.

Tissue and cell expression of TRPV4. Tissue and cell expressions of the TRPV4 protein were studied using specific antibodies in transverse sections of adult mouse retinas and rat vestibular ganglions in RGCs and VGCs isolated from neonate rodent tissues as well as in adult human retina (Fig. 6). In the adult mouse retina, TRPV4 immunostaining was found in the RGCs and the inner plexiform layers (Fig. 6A). RGCs displayed similar TRPV4 protein expression at membrane level in the cell cytosol and neurites in isolated neonate RGCs (Fig. $6 B$ ). In adult human retina (Fig. 6C), RGCs also exhibited TRPV4 immunostaining, suggesting a conserved expression of TRPV4 in RGCs across species. In the vestibular ganglion, TRPV4 immunostaining was observed in the cytosol and in the periphery of the soma, suggesting membrane expression of the TRPV4 channels (Fig. 6D). On isolated neonate VGCs, TRPV4 expression seems to be restricted to the cell bodies, as neuritis were not immunolabeled (Fig. 6E).

Local temperature changes evoked by the laser irradiation. To assess the ability of the laser irradiations to alter significantly the temperature of the bath we measured, in a bath without neurons, the fluorescence variations of LDS 698, a temperaturesensitive dye, on laser irradiations in the range of those that triggered LEVV in sensory neurons. Laser irradiations of $20 \mathrm{~J} / \mathrm{cm}^{2}$ (i.e., 7-ms pulse duration) evoked mean temperature variations of $10 \pm 2^{\circ} \mathrm{C}(n=10)$. This confirmed that the local temperature jumps evoked by the IR laser irradiation were within the activation range of the TRPV4 channels (Güler et al. 2002; Watanabe et al. 2002).

\section{DISCUSSION}

Present data directly demonstrate that the neuronal responses observed in the RGCs and VGCs on IR laser irradiation originates from TRPV4 channel activation. In addition, we show that TRPV4 channels are expressed in the two types of sensory neurons in our culture preparation as well as in adult tissues. Finally, we confirm that the local temperature jumps evoked by the IR laser irradiation are suitable for the activation of the TRPV4 channels. 
A

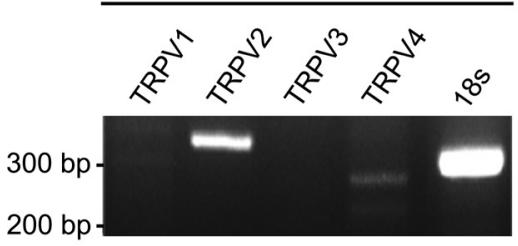

B

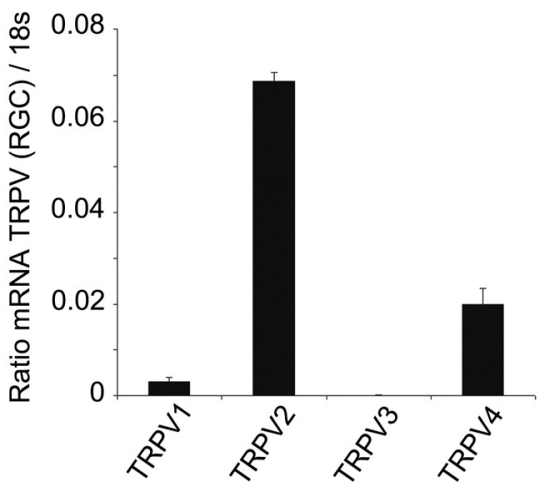

C

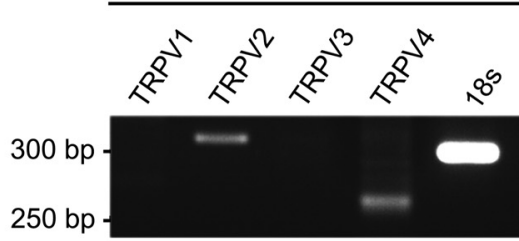

D

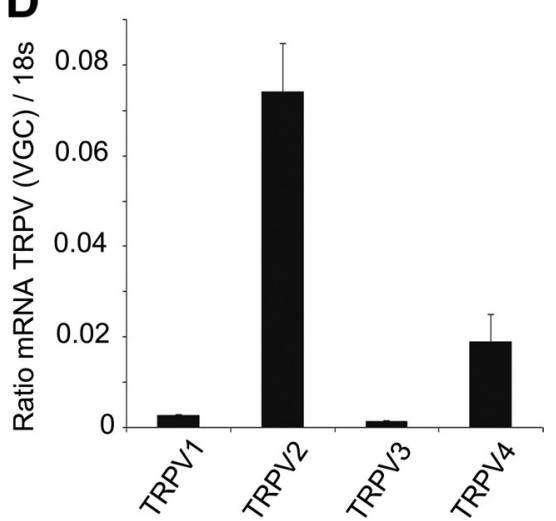

Fig. 4. Expression and quantification of transient receptor potential vanilloid (TRPV) 1-4 channel transcripts in primary cell cultures of sensory neurons. RT-PCR $(A$ and $C)$ and real-time PCR $(B$ and $D)$ of mouse RGCs, 10 days in vitro (10 div; $A$ and $B$ ), and rat VGCs, $6 \operatorname{div}(C$ and $D$ ), both reveal major expression of TRPV2 and TRPV4 mRNAs. Amplifications were normalized to $18 \mathrm{~s}$ ribosomal RNA levels. Each RT-PCR and real-time PCR was performed twice using 1 culture of RGCs or VGCs prepared from 15 mice or 5 rats, respectively.
TRPV channels, besides their sensitivity to vanilloids, also display temperature sensitivity. The sequence of activation of the TRPV channels according to their heat threshold reported in expression systems such as HEK 293 cells (Watanabe et al. 2002) and Xenopus oocytes (Güler et al. 2002) is as follows: TRPV4 $>27^{\circ} \mathrm{C}$, TRPV $3>32^{\circ} \mathrm{C}$, TRPV $1>43^{\circ} \mathrm{C}$, and TRPV2 $>53^{\circ} \mathrm{C}$ (Caterina 2007; Noël et al. 2009). In our preparation, the mean temperature reached during the laser irradiation (37 $\left.\pm 2{ }^{\circ} \mathrm{C} ; n=10\right)$ was above the TRPV4 activation threshold, therefore supporting a thermal activation of the TRPV4 channels. Complete demonstration will, however, require measuring the effective temperature change at the cell membrane level during the laser exposure. Other means of cell activation such as mechanical stimulation or photoactivation are unlikely to take place. Under the present paradigm, no movement of the recorded cells or of the surrounding bathing medium could be seen under visual control. Photoactivation would require the expression of photosensitive pigments. ipRGCs containing melanopsin have been shown to represent $1-2 \%$ of the mammal RGCs in rodents and 0.2\% in humans (Dacey et al. 2005; Do and Yau 2010). Although it cannot be excluded that some ipRGCs may have been included in the recorded cells, the action spectrum of melanopsin $\left(\lambda_{\max } \approx 480 \mathrm{~nm}\right.$; Qiu et al. 2005 ) prevents any photoactivation by the IR laser wavelength used in our study. Moreover, as shown by the lack of effect of 2-APB and $\mathrm{Gd}^{3+}$, the TRPC pathway reported to mediate cell excitations in the ipRGCs (Hartwick et al. 2007) does not contribute to the IR LEVV in the RGCs and VGCs.

Although our pharmacological data designate TRPV4 channels as the main effectors in the induction of LEVV, the involvement of other TRPV channels can be discussed. TRPV4 is indeed the most prominently expressed TRPV channel in RGCs. Despite a slight expression of the TRPV1 mRNAs, this channel type does not contribute to the LEVV, as illustrated by the lack of effect of capsazepine. Likewise, in VGCs, TRPV1 channels that display slight mRNA expression do not contrib- ute to the LEVV. Conversely, TRPV2 and TRPV4 are both strongly expressed in this cell type. Although the TRPV4 channels have been clearly shown to control the LEVV in VGCs and RGCs, we were unable to assess pharmacologically the involvement of TRPV2 in this process because of the lack of specificity of the available TRPV2 blockers. Even though the temperature changes $\left(10 \pm 2^{\circ} \mathrm{C}\right)$ evoked under the present paradigm do not reach the activation threshold $>53^{\circ} \mathrm{C}$ for TRPV2 (Caterina 2007; Noël et al. 2009), it cannot be ruled out that higher temperature elevations would activate this channel subtype. Generally, it can be assumed that any TRPV channels would contribute to the IR laser-evoked responses as long as the temperature changes at the cell membrane reach their respective activation threshold and that they are sufficiently expressed at the cell membrane to alter efficiently the membrane voltage on activation. Although we verified that TRPV4 channels are expressed both by neonate and adult RGCs and VGCs, expression of TRPV1-2 in RGCs varies during development (Leonelli et al. 2009). Although scarcely present in neonate RGCs, they are both strongly expressed in the later phase of retinal development in the RGC layer. Therefore, it cannot be excluded that the expression of these additional TRPV channel types may enhance the excitation range of the RGCs to local temperature changes. However, presently, no evidence suggesting involvement of TRPV channels other than TRPV4 in laser-induced biological responses of sensory neurons has been reported.

Our findings that TRPV4 channels are essential to the induction of LEVVs and that specific voltage-gated calcium and sodium channel blockers abolish the spikelike component of the response allow us to propose a cellular cascade for the LEVV (Fig. 7): on activation, TRPV4 channels allow calcium influx to the cell cytosol. This cation entry drives a membrane depolarization suitable to activate low-threshold voltage-gated calcium channels, leading to further depolarization and subsequent activation of voltage-gated sodium channels. Altogether, 
A
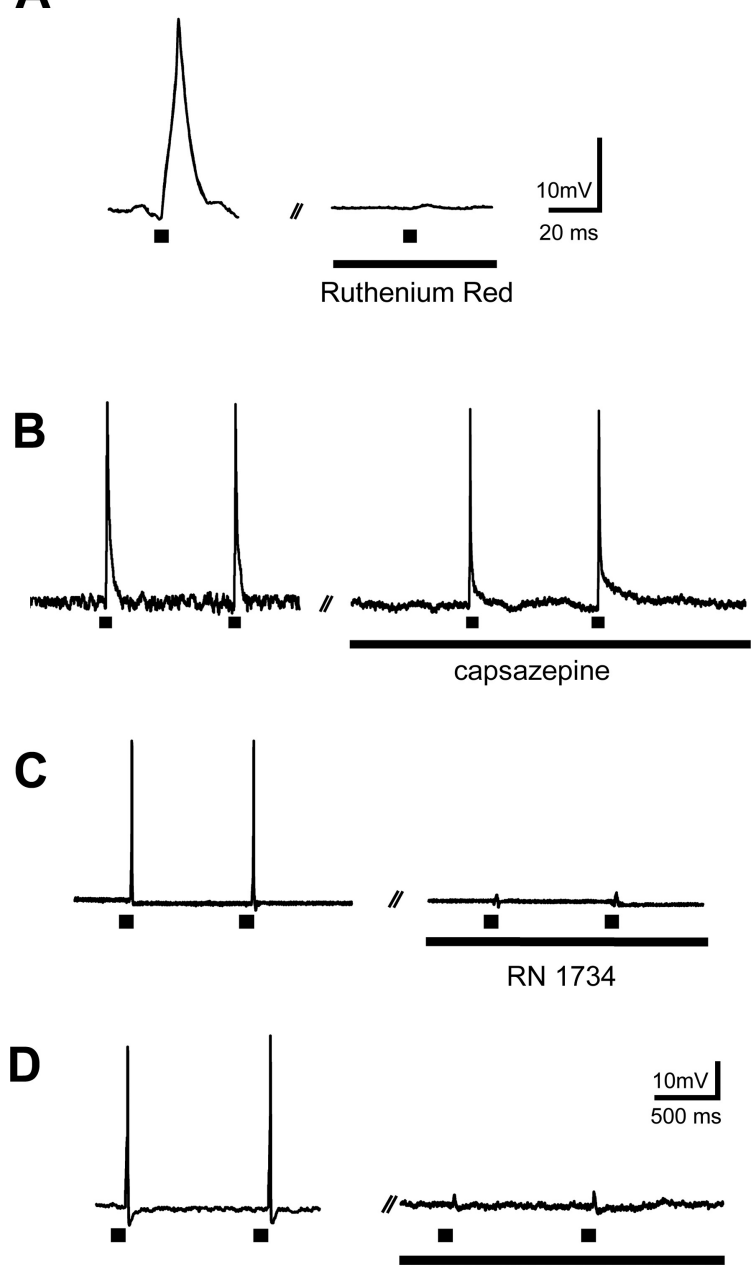

RN 1734

Fig. 5. TRPV4 channels mediate the LEVV in sensory neurons. Each panel is composed of LEVV traces in control saline solution (left traces) and following application of the indicated tested drugs (right traces). A: illustration of the complete block of the LEVV by ruthenium red $(10 \mu \mathrm{M})$, a general TRPV channel blocker in a VGC ( $n=3$ RGCs and 5 VGCs). $B$ : capsazepine (10 $\mu \mathrm{M})$, a TRPV1 channel blocker, does not affect LEVV in a RGC ( $n=2$ RGCs and 4 VGCs). C: RN $1734(5.2 \mu \mathrm{M})$, a specific TRPV4 channel blocker, completely abolishes the LEVV in RGCs $(n=4)$. D: RN $1734(3.2 \mu \mathrm{M})$ prevents the LEVV in VGCs $(n=7)$. Scale in $D$ applies to $B-D$.

this sequence of events allows generation of sodium-based APs in the cells forming the optic and vestibular nerves following IR laser stimulation. Given that expression of ion channels in VGCs is heterogeneous (Chabbert et al. 2001; Kalluri et al. 2010), the small prespike hyperpolarization in a fraction of recorded VGCs likely reflects a different ion channel composition in this subpopulation. Because of minimal species differences in TRPV4 amino acid sequences (Nilius et al. 2004) and the similar TRPV4 protein expression in rodent and adult human RGCs, it is likely that the TRPV4-based process of laser-evoked cell response in these cells could apply to human tissue. The apparent innocuousness of IR lasers for the stimulation of primary sensory neurons in culture suggests that IR laser irradiation, at least in range of the wavelengths and laser power used in the present study, may be a convenient way to stimulate properly these sensory ganglion cells in a prosthesis approach. However, although the present study indicates that sensory neurons can be repetitively irradiated without alteration of their passive membrane properties, the long-lasting consequences of repetitive high-rate stimulations will need to be explored further. Demonstration that excitable cells expressing TRPV channels may be targeted by local and controlled temperature changes also opens novel technical possibilities, specifically regarding the development of nanotechnology. Recent elegant experiments have indeed demonstrated that changes in the membrane potential of hippocampal neurons that express TRPV1 could be achieved through magnetic-field heating of nanoparticles (Huang et al. 2010). Bringing the in vivo proof of concept of the feasibility to excite neurons using IR laser excitation will require solving some important questions such as how to define the optimal location for irradiation of target cells. The irregular expression of TRPV channels along the neuron soma (illustrated by the selective expression of TRPV4 at the soma of adult rat VGCs) as well as the fact that some sensory neurons (vestibular and auditory ganglion cells) are myelinated can also influence the sensitivity of the sensory neurons to IR laser stimulation. In any case, the present demonstration should open novel therapeutic opportunities, particularly in the field of the sensory neuroscience, as TRPV4 channels have now been demonstrated in retinal, vestibular, and auditory ganglion cells (Shen et al. 2006).

In previous experiments on cardiomyocytes, Dittami and colleagues (2011) brought evidence that IR irradiation (in the same energy range of that used in present study) may also modulate the calcium mitochondrial uniporter (mCU). Brief and repetitive IR irradiations significantly increased cytosolic calcium in these cells. Subsequently, the same research group proposed that a similar mechanism may support the IR stimulation of vestibular hair cells that led to excitation of the vestibular afferents in the Toadfish crista ampullaris (Rajguru et al. 2011). Following this interesting observation, it cannot be discarded that the mitochondrial pathway may also be activated in the sensory neurons on IR irradiation. However, it has to be noticed that under present experimental conditions, the removal of the external calcium was sufficient to abolish the LEVV, although a residual depolarizing component remained. This suggests that any IR activation of the mCU may modulate but not be sufficient to drive the LEVV of sensory neurons. Moreover, IR irradiations that activated the hair cells in the experiments of Rajguru et al. (2011) were inefficient on the vestibular afferents. This also suggests that the mechanisms supporting the biological responses to the IR irradiation may differ depending on the cell type. Additional investigations using calcium microfluorimetry technique should allow assessment of the respective weight of these two cellular pathways in the induction of the LEVV. In the same way, investigations using the voltage-clamp configuration of the patch-clamp technique should allow further investigation of the ionic nature of the current that flows through the TRPV4 channel during the laser irradiation. This will help to test whether different channel types participate to the neuron response or to gather indications on the voltage sensitivity of the TRPV4 channel in sensory neurons.

\section{ACKNOWLEDGMENTS}

We thank Dr. M. Pequignot for supplying human retinas (obtained from Dr. Gilles Thuret, Laboratoire Biologie Ingénierie et Imagerie de la Greffe de Cornée). We also thank Drs. C. Delettre, O. Payet, and H. Boukhaddaoui for 

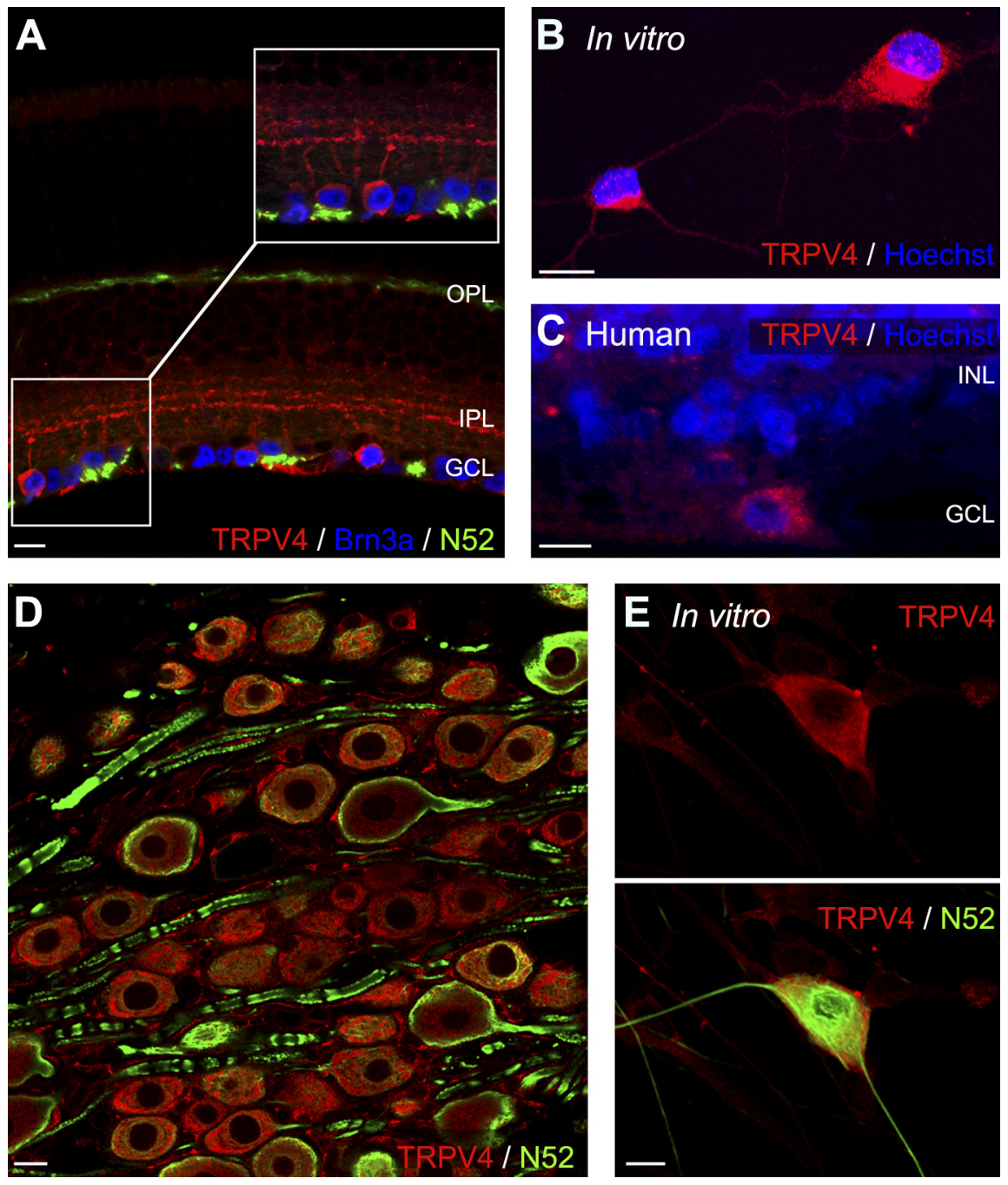

expert technical assistance and Dr. J. Dyhrfjeld-Johnsen for helpful comments on the paper.

\section{GRANTS}

This work was supported by grants from European Aeronautic Defence and Space (EADS), Fédération des Aveugles et Handicapés Visuels de France (FAF), and Union Nationale des Aveugles et Déficients Visuels (UNADEV).

\section{DISCLOSURES}

No conflicts of interest, financial or otherwise, are declared by the author(s).

\section{AUTHOR CONTRIBUTIONS}

E.S.A., J.M.B., G.D., K.C., C.T., S.G., and F.B. performed experiments; E.S.A., G.D., S.G., and F.B. analyzed data; E.S.A., J.M.B., S.G., F.B., A.M., and C.C. interpreted results of experiments; E.S.A., G.D., S.G., and C.C.

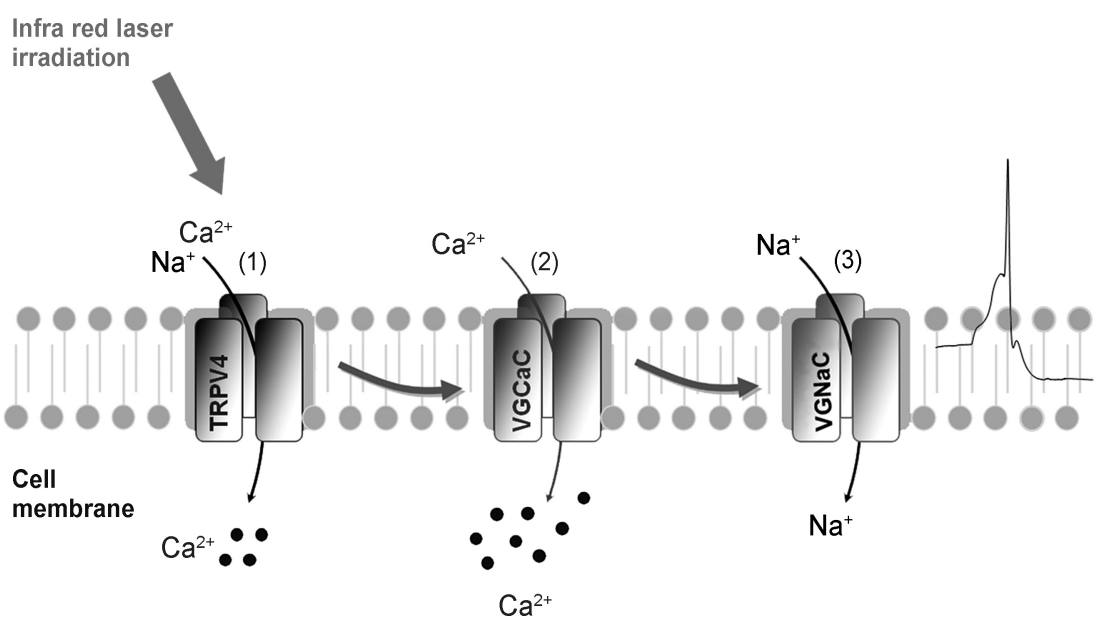

Fig. 6. Immunolocalization of TRPV4 using specific antibodies in slices of 2-mo mouse retina $(A)$, RGCs [postnatal days 2-4 (P2-P4) animals, 5 div, 3 experiand human RGC (C;2 als also demonstrated in 2-mo Wistar VGC P5 animals ( $E ; 3$ experiments) without and with N52 staining. TRPV4 (red), N52 (green), Brn3a, a specific RGC transcriptional factor, and Hoechst (blue) are shown. Scale bar of $10 \mu \mathrm{m}$ applies to all. OPL, outer plexiform layer; IPL, inner plexiform layer; GCL, ganglion cell layer; INL, inner nuclear layer.
Fig. 7. Proposed sequence of events in the induction of the neuronal response to the IR laser irradiation into sensory neurons. Supraliminal thermal stimulation opens the TRPV4 channels allowing a calcium influx into the cell cytosol (1) that in turn will lead to a membrane depolarization resulting in an activation of the voltage-gated $\mathrm{Ni} / \mathrm{Cd}$-sensitive calcium channels (2). Additional calcium influx in the cell will increase the membrane depolarization that will lead to the generation of sodium-based action potentials in the sensory neurons through activation of voltage-gated (TTX-sensitive) sodium channels (3). 
prepared figures; E.S.A., I.M., M.D., G.L., C.H., A.M., and C.C. drafted manuscript; E.S.A. and C.C. approved final version of manuscript; C.C. conception and design of research; C.C. edited and revised manuscript.

\section{REFERENCES}

Angelico P, Testa R. TRPV4 as a target for bladder overactivity. F1000 Biol Rep 2: 12, 2010.

Brugeaud A, Travo C, Dememes D, Lenoir M, Llorens J, Puel JL, Chabbert C. Control of hair cell excitability by vestibular primary sensory neurons. J Neurosci 27: 3503-3511, 2007.

Caterina MJ. Transient receptor potential ion channels as participants in thermosensation and thermoregulation. Am J Physiol Regul Integr Comp Physiol 292: R64-R76, 2007.

Chabbert C, Chambard JM, Valmier J, Sans A, Desmadryl G. Hyperpolarization-activated (Ih) current in mouse vestibular primary neurons. Neuroreport 12: 2701-2704, 2001.

Chambard JM, Chabbert C, Sans A, Desmadryl G. Developmental changes in low and high voltage-activated calcium currents in acutely isolated mouse vestibular neurons. J Physiol 518: 141-149, 1999.

Chen Y, Bedell M, Zhang K. Age-related macular degeneration: genetic and environmental factors of disease. Mol Interv 10: 271-281, 2010.

Colodetti L, Weiland JD, Colodetti S, Ray A, Seiler MJ, Hinton DR, Humayun MS. Pathology of damaging electrical stimulation in the retina. Exp Eye Res 85: 23-33, 2007.

Coppeta J, Rogers C. Dual emission laser-induced fluorescence for direct planar scalar behavior measurements. Exp Fluids 25: 1-15, 1998.

Dacey DM, Liao HW, Peterson BB, Robinson FR, Smith VC, Pokorny J, Yau KW, Gamlin PD. Melanopsin-expressing ganglion cells in primate retina signal colour and irradiance and project to the LGN. Nature 433: 749-754, 2005.

Dayanithi G, Desmadryl G, Travo C, Chabbert C, Sans A. Trimetazidine modulates AMPA/kainate receptors in rat vestibular ganglion neurons. Eur J Pharmacol 574: 8-14, 2007.

Dittami GM, Rajguru SM, Lasher RA, Hitchcock RW, Rabbitt RD. Intracellular calcium transients evoked by pulsed infrared radiation in neonatal cardiomyocytes. J Physiol 589: 1295-1306, 2011.

Do MT, Yau KW. Intrinsically photosensitive retinal ganglion cells. Physiol Rev 90: 1547-1581, 2010.

Fork RL. Laser stimulation of nerve cells in Aplysia. Science 171: 907-908, 1971

Fried NM, Lagoda GA, Scott NJ, Su LM, Burnett AL. Laser stimulation of the cavernous nerves in the rat prostate, in vivo: optimization of wavelength, pulse energy, and pulse repetition rate. Conf Proc IEEE Eng Med Biol Soc 2008: 2777-2780, 2008.

Güler AD, Lee H, Iida T, Shimizu I, Tominaga M, Caterina M. Heatevoked activation of the ion channel, TRPV4. J Neurosci 22: 6408-6414, 2002.

Hartwick AT, Bramley JR, Yu J, Stevens KT, Allen CN, Baldridge WH, Sollars PJ, Pickard GE. Light-evoked calcium responses of isolated melanopsin-expressing retinal ganglion cells. J Neurosci 27: 13468-13480, 2007.

Haynes DS, Young JA, Wanna GB, Glasscock ME 3rd. Middle ear implantable hearing devices: an overview. Trends Amplif 13: 206-214, 2009.

Huang H, Delikanli S, Zeng H, Ferkey DM, Pralle A. Remote control of ion channels and neurons through magnetic-field heating of nanoparticles. Nat Nanotechnol 5: 602-606, 2010.

Izzo AD, Richter CP, Jansen ED, Walsh JT Jr. Laser stimulation of the auditory nerve. Lasers Surg Med 38: 745-753, 2006.
Izzo AD, Walsh JT Jr, Ralph H, Webb J, Bendett M, Wells J, Richter CP. Laser stimulation of auditory neurons: effect of shorter pulse duration and penetration depth. Biophys J 94: 3159-3166, 2008.

Kalluri R, Xue J, Eatock RA. Ion channels set spike timing regularity of mammalian vestibular afferent neurons. J Neurophysiol 104: 2034-2051, 2010.

Kamei S, Chen-Kuo-Chang M, Cazevieille C, Lenaers G, Olichon A, Bélenguer P, Roussignol G, Renard N, Eybalin M, Michelin A, Delettre C, Brabet P, Hamel CP. Expression of the Opal mitochondrial protein in retinal ganglion cells: its downregulation causes aggregation of the mitochondrial network. Invest Ophthalmol Vis Sci 46: 4288-4294, 2005.

Kim H, Dixit S, Green CJ, Faris GW. Nanodroplet real-time PCR system with laser assisted heating. Opt Express 17: 218-227, 2009.

Kunert-Keil C, Bisping F, Kruger J, Brinkmeier H. Tissue-specific expression of TRP channel genes in the mouse and its variation in three different mouse strains. BMC Genomics 7: 159, 2006.

Leonelli M, Martins DO, Kihara AH, Britto LR. Ontogenetic expression of the vanilloid receptors TRPV1 and TRPV2 in the rat retina. Int $J$ Dev Neurosci 27: 709-718, 2009.

Nilius B, VJ, Prenen J, Droogmans G, Voets T. TRPV4 calcium entry channel: a paradigm for gating diversity. Am J Physiol Cell Physiol 286: C195-C205, 2004.

Noël J, Zimmermann K, Busserolles J, Deval E, Alloui A, Diochot S, Guy $\mathbf{N}$, Borsotto M, Reeh P, Eschalier A, Lazdunski M. The mechanoactivated $\mathrm{K}^{+}$channels TRAAK and TREK-1 control both warm and cold perception. EMBO J 28: 1308-1318, 2009.

Popovic D, Gordon T, Rafuse VF, Prochazka A. Properties of implanted electrodes for functional electrical stimulation. Ann Biomed Eng 19: 303316, 1991.

Qiu X, Kumbalasiri T, Carlson SM, Wong KY, Krishna V, Provencio I, Berson DM. Induction of photosensitivity by heterologous expression of melanopsin. Nature 433: 745-749, 2005.

Rajguru SM, Richter CP, Matic AI, Holstein GR, Highstein SM, Dittami GM, Rabbitt RD. Infrared photostimulation of the crista ampullaris. $J$ Physiol 589: 1283-1294, 2011.

Saihan Z, Webster AR, Luxon L, Bitner-Glindzicz M. Update on Usher syndrome. Curr Opin Neurol 22: 19-27, 2009.

Shen J, Harada N, Kubo N, Liu B, Mizuno A, Suzuki M, Yamashita T. Functional expression of transient receptor potential vanilloid 4 in the mouse cochlea. Neuroreport 17: 135-139, 2006.

Teudt IU, Nevel AE, Izzo AD, Walsh JT Jr, Richter CP. Optical stimulation of the facial nerve: a new monitoring technique? Laryngoscope 117: 16411647, 2007.

Vincent F, Acevedo A, Nguyen MT, Dourado M, DeFalco J, Gustafson A, Spiro P, Emerling DE, Kelly MG, Duncton MA. Identification and characterization of novel TRPV4 modulators. Biochem Biophys Res Commun 389: 490-494, 2009.

Vriens J, Appendino G, Nilius B. Pharmacology of vanilloid transient receptor potential cation channels. Mol Pharmacol 75: 1262-1279, 2009.

Watanabe H, Vriens J, Suh SH, Benham, Droogmans G, Nilius B. Heat activation of TRPV4 channels in HEK 293 cell expression system and in native mouse aorta endothelial cells. J Biol Chem 277: 47044-47051, 2002.

Wells J, Konrad P, Kao C, Jansen ED, Mahadevan-Jansen A. Pulsed laser versus electrical energy for peripheral nerve stimulation. J Neurosci Methods 163: 326-337, 2007a.

Wells J, Kao C, Konrad P, Milner T, Kim J, Mahadevan-Jansen A, Jansen ED. Biophysical mechanisms of transient optical stimulation of peripheral nerve. Biophys J 93: 2567-2580, 2007b.

Wells J, Kao C, Mariappan K, Albea J, Jansen ED, Konrad P, Mahadevan-Jansen A. Optical stimulation of neural tissue in vivo. Opt Lett 30: 504-506, 2005. 